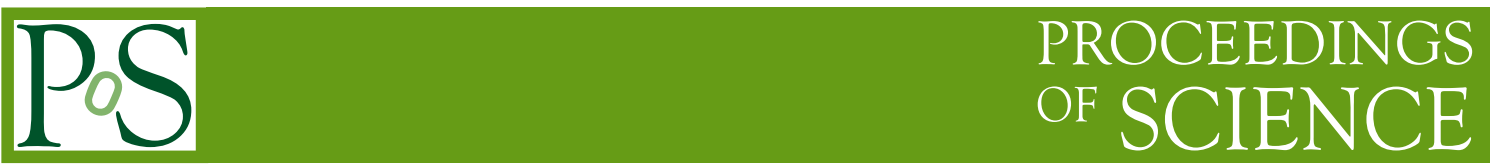

\title{
Recent results on screening masses
}

\author{
Edwin Laermann* with A. Francis, S. Fröhlich and S. Mukherjee \\ Fakultät für Physik, Universität Bielefeld, 33615 Bielefeld, Germany \\ E-mail: edwin@physik.uni-bielefeld.de
}

and the RBC-Bielefeld Collaboration

\begin{abstract}
Recent results for screening masses at high temperature are presented. The spatial correlation functions of operators with meson quantum numbers were obtained on quenched gauge configurations with non-perturbatively improved Wilson quarks as well as, in a different round of analysis, on the $N_{F}=2+1$ configurations generated in the course of RBC-Bielefeld's analysis of the Equation of State. While vector and axial vector channels signal the restoration of chiral symmetry in both cases, in $2+1$ flavor QCD pseudoscalar and isovector scalar become degenerate only at a temperature higher than the chiral crossover one, thus indicating that the $U_{A}(1)$ is effectively restored only at higher temperatures. In the investigated temperature range pseudoscalar and vector screening masses do not become equal, contrary to the free case and to leading order perturbation theory. For the quenched Wilson case the differences could be established in the infinite volume continuumm limit.
\end{abstract}

The XXVI International Symposium on Lattice Field Theory

July 14-19 2008

Williamsburg, Virginia, USA

${ }^{*}$ Speaker. 


\section{Introduction}

One of the goals of finite temperature lattice QCD studies is the analysis of the existence and of the properties of hadronic excitations in the quark-gluon plasma. This information is encoded in the spectral density $\sigma\left(p_{0}, \vec{p}\right)$. Since the temporal extent of high temperature lattices is limited by $1 / T$ (and even advanced analyses of temporal hadron propagators utilizing MEM may have their subtleties) it may not be futile to study also spatial correlation functions (for an account of earlier studies see e.g. [1], a recent investigation based on quenched overlap fermions is presented in [2]). While the spatial correlators $G^{S}(z)$ depend on the spectral density via

$$
G^{S}(z)=\int_{-\infty}^{+\infty} \frac{d p}{2 \pi} e^{i p z} \int_{-\infty}^{+\infty} d p_{0} \frac{\sigma\left(p_{0}, p\right)}{p_{0}}
$$

their expontial decay with $z, G^{S}(z) \sim \exp \left(-m^{s c r} z\right)$ delivers the screening masses $m^{s c r}$ and, moreover, reveals information about the restoration of chiral symmetries in the plasma phase.

In this talk we also address the question of how quickly, at temperatures up to a few hundred $\mathrm{MeV}$, the screening masses approach their infinite temperature, free case limit. This has been computed in the continuum [3], with the result

$$
m_{\mathrm{eff}}^{s c r}(z)=-\frac{1}{G^{S}(z)} \frac{\partial G^{S}(z)}{\partial z} \simeq 2 \pi T\left\{1+\frac{1}{2 \pi T z}+\ldots\right\}
$$

for the effective, $z$-dependent screening mass. The leading order is given by twice the lowest fermionic Matsubara frequency, $2 \pi T$. However, note the slow approach to this value which at given temperature is only reached in the limit $z \rightarrow \infty$. Lowest order perturbative corrections to this result have been given by [4] and have turned out to be positive, $m^{s c r}=2 \pi T\left(1+c g^{2}\right)$ with a small coefficient $c=0.022$ for $N_{F}=0$ and $c=0.033$ for $N_{F}=3$.

In the following, at first results for quenched Wilson quarks are presented, with particular emphasis on the infinite volume limit. We then proceed to full QCD including light as well as strange staggered quarks. Earlier results have already been shown in [5] (Wilson) and in [6] (staggered case).

\section{Screening masses from massless quenched Wilson quarks}

In this section, results for screening masses obtained with Wilson-type quarks in the quenched approximation will be presented. The gauge configurations were generated with a standard plaquette gauge action. For the fermions the non-perturbatively improved Sheikholeslami-Wohlert action [8] was used such that the discretization errors start at $\mathscr{O}\left(a^{2}\right)$. Above the transition temperature $T_{c} \simeq 270 \mathrm{MeV}$ [7] the spatial correlators were computed for massless quarks, i.e. at hopping parameter values corresponding to vanishing pion mass at $T=0$ [9]. The analysis is focussed on temperatures of $1.5 T_{c}$ and $3 T_{c}$. In order to control discretization effects $\sim a T=1 / N_{\tau}$ at both temperatures isotropic lattices with $N_{\tau}=8,12$ and 16 were generated. Finite volume effects are controlled by the aspect ratio $L T=N_{\sigma} / N_{\tau}$ for which we chose 2,3,4 and 8 at each temperature and each $N_{\tau}$. Thus, the largest lattice analyzed was of size $128^{3} \times 16$.

An immediate observation, already at the level of correlation functions, concerns the restoration of chiral $S U_{L}\left(N_{F}\right) \otimes S U_{R}\left(N_{F}\right)$ symmetry at $1.2 T_{c}$, reflected in the degeneracy of the (transverse) 

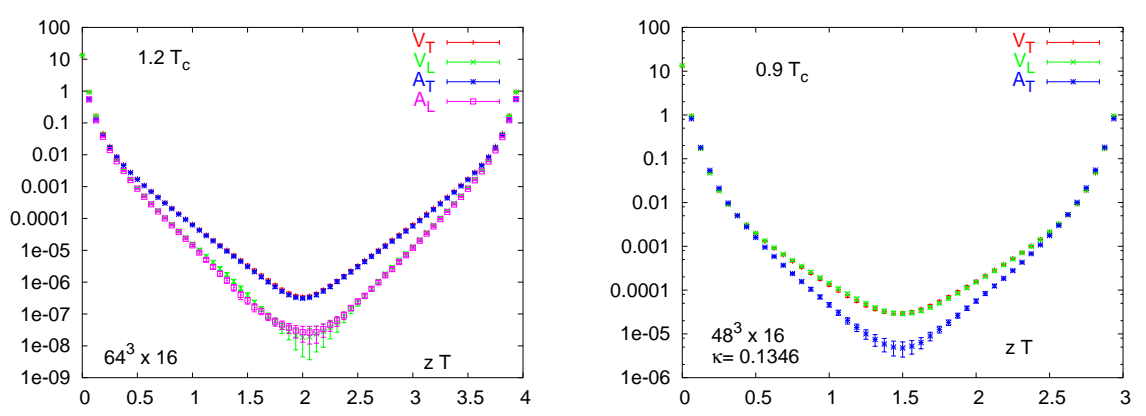

Figure 1: Transverse $(\mathrm{T})$ and longitudinal $(\mathrm{L})$ vector $(\mathrm{V})$ and axial vector $(\mathrm{A})$ spatial correlation functions for quenched Wilson fermions above and below $T_{c}$.

vector $\left(V_{T}\right)$ and axial vector $\left(A_{T}\right)$ channel, Fig. 1 (left). Due to the loss of Lorentz invariance at finite temperature, as has been emphasized by [10], the longitudinal components $\left(V_{L}\right.$ and $\left.A_{L}\right)$ of the spatial correlators, while being degenerate with each other, are different from the transverse ones. However, this splitting between transverse and longitudinal polarizations only occurs above $T_{c}$, Fig. 1 (right), while the axial vector remains to be heavier than the vector at temperatures below $T_{c}$, at least up to $0.9 T_{c}$.

In order to address the systematic errors arising from finite volume and finite lattice spacing we first computed the screening masses in the free, infinite temperature limit on lattices of various size. In Fig. 2 (left) we plot the effective pion screening mass, $m_{\mathrm{eff}}(z) \equiv m_{\mathrm{eff}}^{\text {scr }}(z)$, as a function of $1 /(z T)$. On the lattice, to be definite, we chose $z T=0.25 N_{\sigma} / N_{\tau}$, thereby introducing the aspect ratio as the controlling parameter. As becomes clear from Fig. 2 (left), in the continuum as well as on the lattice finite volumes ( $z$-values) drive the screening masses up, with a leading behavior $\sim 1 /(L T)$. On the contrary, finite lattice spacing effects push the screening masses below the continuum values, Fig. 2 (right). The free case value of $2 \pi T$, in the continuum as well as on the lattice, is only reached at infinite separation, $1 / z \rightarrow 0$.
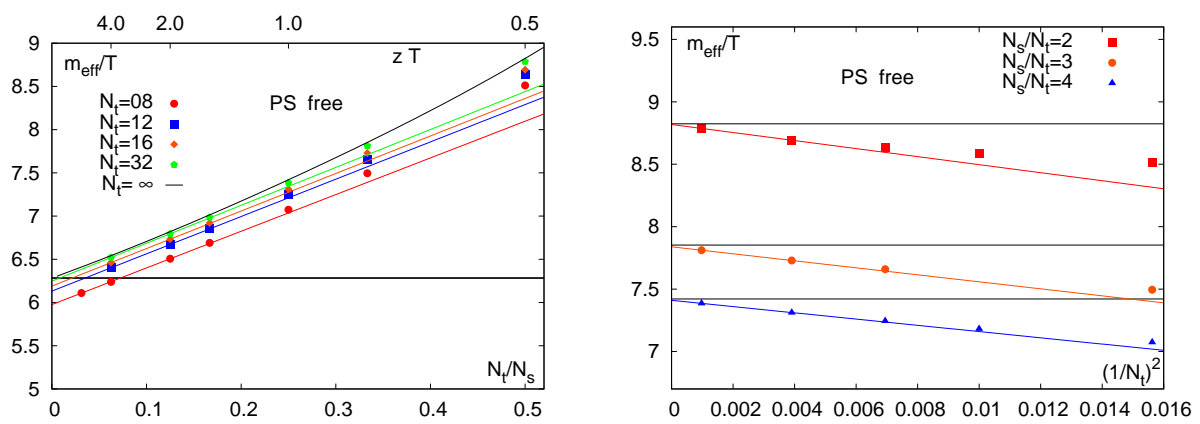

Figure 2: Pion screening masses in the free, infinite temperature limit. The continuum results (black lines) are compared to quenched Wilson data from lattices with various aspect ratios and $N_{\tau}$ values as indicated.

Qualitatively, the same picture as at infinite temperature emerges in the interacting case at temperatures $1.5 T_{c}$ and $3 T_{c}$, Fig. 3. As true for many observables, in the interacting theory the finite volume effects are less severe than in the free case. Also, the slope in $1 /(L T)$, Fig. 3 (left), is 

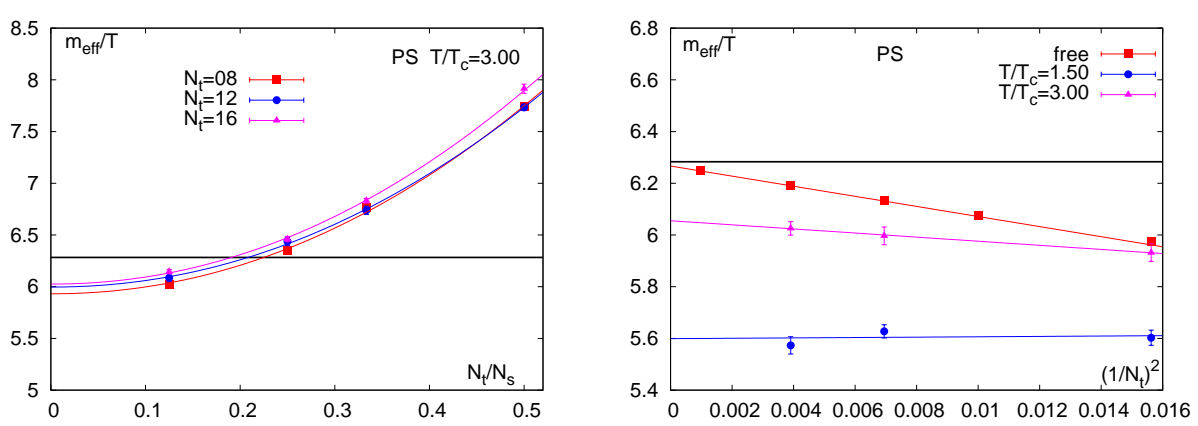

Figure 3: Pseudoscalar screening masses at $3 T_{c}$ (left) as a function of the inverse aspect ratio, for three $N_{\tau}$ values as indicated, together with the fit results as explained in the text. Right: Continuum limit extrapolations of the (infinite volume extrapolated) pion screening masses at $1.5 T_{c}$ and $3 T_{c}$, compared with the free case extrapolation (red line). The horizontal black lines (left and right) indicate the free case value $2 \pi$.

not linear: fitting the data for $m^{s c r} / T$ to the behavior $A+B\left(N_{\tau} / N_{\sigma}\right)^{p}$ where the power $p$ is left as a free parameter, returns $p=2.2(1)$ at $1.5 T_{c}$ and a slightly less value of 2.05(10) at $3 T_{c}$, for both pseudoscalar and vector. Turning to the discretization effects, Fig. 3 (right), they are proportional to $(a T)^{2}=\left(1 / N_{\tau}\right)^{2}$, as they should because a non-perturbatively $\mathscr{O}\left(a^{2}\right)$ improved action has been used. Compared to the free case, red line in Fig. 3 (right), however, the slopes are smaller.

Although the pion screening mass is rising with temperature, at the temperatures investigated, which reach about $800 \mathrm{MeV}$, there remain significant differences between the pseudoscalar screening mass and its free value $2 \pi T$. These differences have now been established in the infinite volume continuum limit.

\section{Screening masses in full QCD with light staggered quarks}

The results presented in this section are based on the gauge configurations generated for the Equation-of-State analysis of the RBC-Bielefeld collaboration [11]. They cover a temperature range between 140 and about $440 \mathrm{MeV}$ and originate from lattices with temporal extents $N_{\tau}=4,6$ and 8 and aspect ratio $N_{\sigma} / N_{\tau}=4$. For the quarks an improved p4fat3 action was used for two light and one strange quark, respectively. The quark masses were tuned such that at $T=0$ the kaon achieves its physical mass and $m_{\pi}=220 \mathrm{MeV}$.

As for the quenched Wilson case, finite volume as well as discretization effects were first computed in the infinite temperature limit. These results [6] turn out to qualitatively follow the Wilson case: finite volumes increase the screening masses and lattice spacing effects decrease them.

We have analyzed point-point correlations for local meson operators built from light and strange quarks in all flavor combinations. At temperatures below about $200 \mathrm{MeV}$, we unfortunately had to observe that the signals in the vector and axial vector channels are too noisy to reliably extract a screening mass. This is presumably due to, first, poor overlap properties of point operators to mesonic bound states populating the low temperature phase of QCD as has been noted at zero temperature. And second, at low temperature the local vector and axial vector operators also project onto so-called parity partners which are not easy to disentangle. At higher tempera- 
tures these contributions vanish rather rapidly, leading to a correlation function which is dominated by one (screening) state and its excitations. Indeed, above about $200 \mathrm{MeV}$ we are able to extract screening masses for one transverse vector as well as one transverse axial vector. Fig. 4 shows that both become equal within errors. This holds even in the light-strange sector. In the figure results from all three different $N_{\tau}$ lattices, i.e. different lattice spacings are shown for the light-light sector. The data reveal that the discretization effects are present but small. Guided by the comparison between free and interacting Wilson case we expect finite volume effects to increase the screening masses not only in the staggered free but also interacting case. The coincidence with the free continuum value of $2 \pi T$ as exhibited in Fig. 4 may thus be accidental.
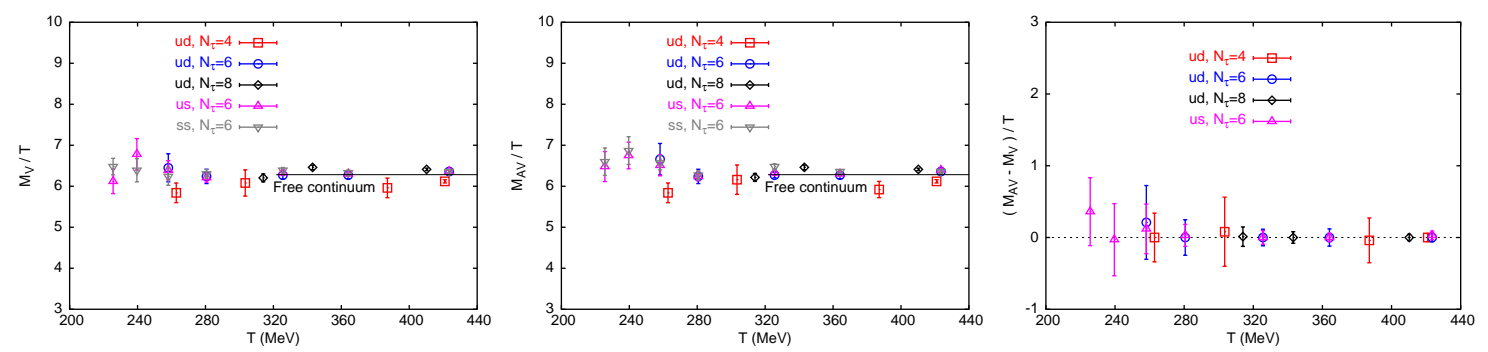

Figure 4: Transverse vector (left) and axial vector (middle) screening masses and their difference (right) for light-light, light-strange and strange-strange flavor combinations in $2+1$ flavor QCD with an improved p4fat3 quark action.

In Fig. 5 we show the results for the pseudoscalar and isovector scalar channels. At low temperature the pion screening mass stays approximately constant (and equal to its zero temperature mass [6]) before experiencing a rapid increase at about $200 \mathrm{MeV}$, followed by a slow approach towards the free value. A qualitatively similar yet much less pronounced behavior is also seen in the kaon and the strange-strange flavor combination. At the same temperature of about 200 $\mathrm{MeV}$ the light-light scalar develops a marked minimum before also approaching slowly the free value. Despite showing a drastic change of behavior simultaneously, pseudoscalar and scalar become degenerate only at temperatures above about $250 \mathrm{MeV}$. Although the chiral limit is not yet reached this observation suggests that the anomalous $U_{A}(1)$ symmetry is not effectively restored at the chiral crossover temperature.

Discretization effects for the (pseudo) scalars are somewhat larger than in the (axial) vector
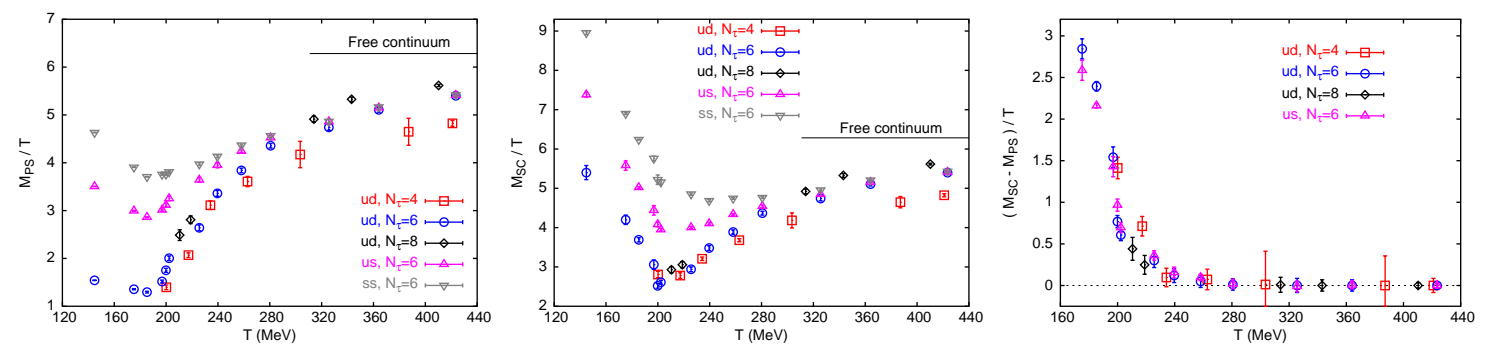

Figure 5: Pseudo scalar (left) and scalar (middle) screening masses and their difference (right) in $2+1$ flavor QCD. As in Fig. 4, for the light-light flavor combination results from all three lattice spacing sets are shown. 

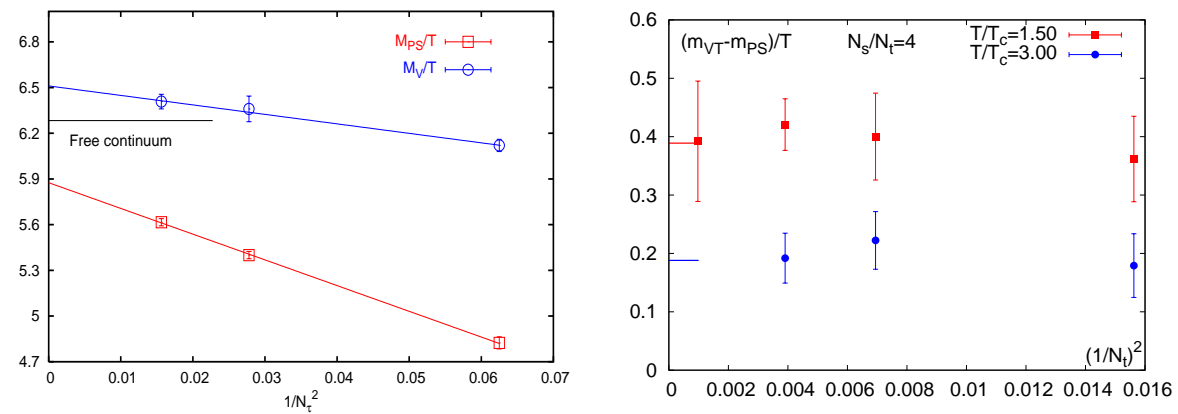

Figure 6: Left: Continuum extrapolation for vector and pseudoscalar screening mass in $2+1$ flavor QCD at $T \simeq 420 \mathrm{MeV}$ and at fixed aspect ratio $N_{\sigma} / N_{\tau}=4$. Right: The difference between vector and pseudoscalar screening masses from quenched Wilson fermions as a function of the lattice spacing at the same fixed aspect ratio of 4 . The horizontal short lines denote the infinite volume continuum limit at the two temperatures considered.

channels, in particular at large $T$, see Fig. 6 (left). Here, the data at a temperature of roughly 420 $\mathrm{MeV}$ are extrapolated to the continuum limit, both for the vector and the pseudoscalar. Even in the continuum limit, there is a significant difference between both screening masses at this temperature. Although the data have been obtained at fixed aspect ratio 4, and the corresponding presumably accidental coincidence of the vector with the continuum free result has been commented on already, this difference appears very unlikely to vanish in the infinite volume limit. For comparison, in Fig. 6 (right) we show the difference between vector and pseudoscalar at the same aspect ratio for quenched Wilson fermions. Apart from the nearly independence on the lattice spacing the figure shows that the pseudoscalar-vector difference is also not much affected by finite volume, note in particular the infinite volume continuum extrapolations indicated by the short horizontal lines at the left hand border. On the other hand, the difference clearly is temperature dependent and decreases when the temperature is raised.

\section{Summary}

For both quenched as well as $2+1$ flavor QCD, in the investigated temperature range, i.e. up to about $800 \mathrm{MeV}$ for the quenched case and about $420 \mathrm{MeV}$ for full QCD, there remain significant differences between pseudoscalar and vector screening masses and thus between the screening masses in this temperature regime and the predictions of free as well as leading order perturbation theory in the high temperature limit. For the quenched case these deviations could be established in the infinite volume continuum limit.

\section{Acknowledgements}

We thank S. Shcheredin and S. Wissel for their contributions at an earlier stage of the project. Apart from apeNEXT (Bielefeld) and QCDOC as well as NYblue (BNL) resources the analysis profited from access to NIC facilities at the FZ Jülich. We thank all involved operating staff. 


\section{References}

[1] F. Karsch and E. Laermann, in Quark-Gluon Plasma III (R. Hwa, ed.).

[2] R. Gavai, S. Gupta and R. Lacaze, arXiv:0803.1368[hep-lat].

[3] W. Florkowski and B. Friman, Z. Phys. A347 (1994) 271.

[4] M. Laine and M. Vepsäläinen, JHEP 402 (2004) 4.

[5] S. Wissel, PoS (LAT2005) 164.

[6] S. Mukherjee, PoS (LAT2007) 210.

[7] G.Boyd et al., Nucl. Phys. B469 (1996) 419.

[8] B. Sheikholeslami and R. Wohlert, Nucl. Phys. B259 (1985) 572;

M. Lüscher et al., Nucl. Phys. B491 (1997) 323.

[9] M. Lüscher et al., Nucl. Phys. B491 (1997) 344.

[10] S. Gupta, Phys. Rev. D60 (1999) 094505.

[11] M. Cheng et al. (RBC-Bielefeld coll.), Phys. Rev. D77 (2008) 014511. 